\title{
Design of Power Supply for Three-Phase Bearingless Switched Reluctance Motor Based On Spwm
}

\author{
Wang Hao, Hu Danfeng* \\ (School of Electronics \& Information, Soochow University, China,) \\ No. 1 Shizi Street, School of Electronics \& Information, Soochow University, Suzhou, Jiangsu, China, 215006.
}

\begin{abstract}
Bearingless switched reluctance motor has the advantages of switched reluctance motor such as simple structure, high efficiency and satisfactory robustness and also the inherent high radial force for rotor shaft magnetic suspension. In order to meet the requirements of high speed and stable operation for it, the power supply needs to be well designed. A voltage source inverter is commonly used to supply a three-phase induction motor with variable frequency and variable voltage for variable speed applications. A suitable sine pulse width modulation technique is employed to obtain the required output voltage in the line side of the inverter. The principle, hardware circuit and MCU program are introduced. The result shows the power supply satisfies the demand of BSRM.
\end{abstract}

Keywords: SPWM, Bearingless switched reluctance motor, Power supply, dsPIC30F2010, IR2110.

\section{Introduction}

BSRM (Bearingless Switched Reluctance Motor) is a novel type of reluctance motors which applied magnetic suspension technology on the traditional ones [1-2]. Switched reluctance motors are well known for their vibration and noise caused by high radial force. Fortunately, the bearingless technology is suitable to solve these problems [3-4]. The BSRMs don't need specially designed mechanical bearings. Instead of those, the windings generate suspending force are added to the stator windings which combine the magnetic fields caused by the two kinds of winding into a whole part and thus the stable suspension and rotation of the rotor can be realized by adjusting the current's magnitude and direction in windings through some control strategies [5]. The winding structure is shown in Fig. 1. BSRM has the advantage of reluctance motor and magnetic suspension motor and a broad perspective in many application area, such as energy, medical and robots.

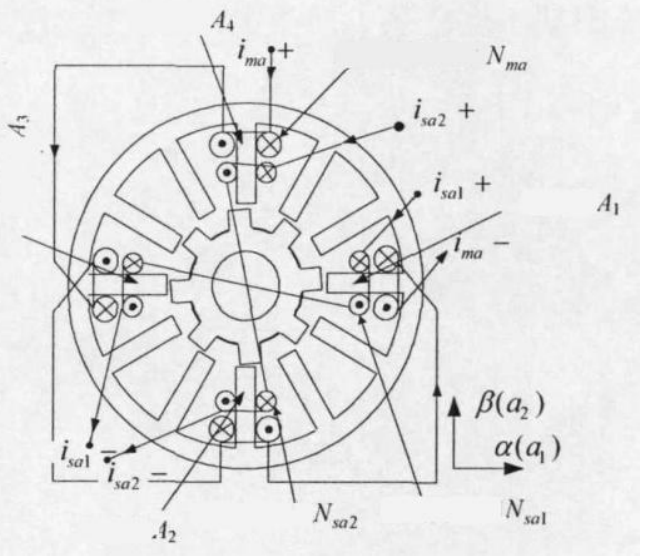

Figure 1. Winding Structure of BSRM

The driving power is the base of realizing stable suspension and rapidly rotation in BSRM. There are three phases in the motor and two coils in each stator tooth which belong to main winding and suspension winding separately. The main winding in each phase is composed of four coils which are at the mutually perpendicular positions in a series connection. The suspension winding $\alpha$ in each phase is composed of two coils which are diametrically opposed in a series connection and the suspension winding $\beta$ is composed of two coils which are diametrically opposed and perpendicular to $\alpha$ in a series connection. The mutual difference of current angles in three-phase winding is $120^{\circ}$. According to the principle of suspension force generating, the current direction in suspension winding is determined by the direction of suspension force needed. So the three-phase fully controlled bridge circuit is adopt in the driving power.

The technique which can decrease the pulsation torque is a key point in the research of BSRM design. Using 
SPWM (Sine wave Pulse Width Modulation) technique in driving power of BSRM is a effective way to achieve this [6-8]. The high frequency and power ultrasonic sinusoidal power supply can make the motor work effectively. Now the hi-power sinusoidal power supplies are limited to the frequency below $400 \mathrm{~Hz}$ and the high frequency inverter limited to the square wave, which fails to meet the demands. In view of the high efficiency of the inverter switch, the great switching loss and the immense output power, the topological structure and the working rationale for the purpose of analyzing and demonstrating the issues such as the hardware construction, the controlling scheme, the parameter selection and the software development on the basis of the SPWM technology are discussed. The design of hardware circuit and MCU(Micro Control Unit) program are also introduced in this article.

\section{Method}

SPWM technique converts the sine wave into small number of pulses which have different width. It produces pulsating waves in which the width of pulses change according to the amplitude of sine wave voltage. For example, the amplitude of voltage is minimum at the start of sine wave at 0 degree and then it starts increasing up until reaches at 90 degree. After that, amplitude start decreasing again with reverse fashion as it is increased. In SPWM technique we follow the same procedure to make pulsating wave signal in which width of each pulse varied according to same fashion as follow by sine wave magnitude. In the most straightforward implementation, generation of the desired output voltage is achieved by comparing the desired reference waveform (modulating signal) with a high-frequency triangular carrier' wave as depicted schematically in Fig. 2. Depending on whether the signal voltage is larger or smaller than the carrier waveform, either the positive or negative DC (Direct Current) voltage is applied at the output. Over the period of one triangle wave, the average voltage applied to the load is proportional to the amplitude of the signal (assumed constant) during this period. The resulting chopped square waveform contains a replica of the desired waveform in its low frequency components, with the higher frequency components being at frequencies of an close to the carrier frequency. The root mean square value of the AC (Alternating Current) voltage waveform is still equal to the DC voltage, and hence the total harmonic distortion is not affected by the PWM process. The harmonic components are merely shifted into the higher frequency range and are automatically filtered due to inductances in the $\mathrm{AC}$ system.

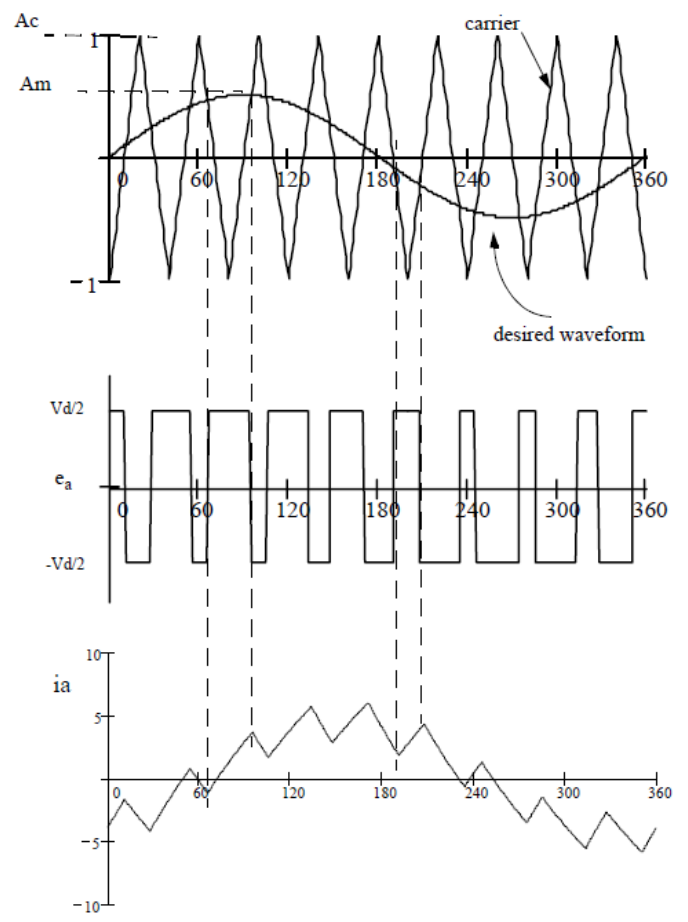

Figure 2. Principal of Pulse Width Modulation

\section{There are commonly two ways to generate SPWM:}

(1) By comparing reference sinusoidal wave with triangular carrier wave of frequency $f_{c}$. In this method, frequency of reference sinusoidal signal is the frequency of sine wave output. This can be implemented by analog electronic components. 
(2) By using MCU or other digital electronic components. This method is preferable because it is cheaper and easy to implement. The MCU can also be used for other functions like protection, digital display of current and voltage and communication with main controller.

We adopt asymmetrical half bridge converter circuit in the design of power supply for three phase BSRM. The diagram of it is shown in Fig. 3. The $L_{A m}, L_{B m}$ and $L_{C m}$ behalf the three windings in BSRM. The $V D_{1} \sim V D_{6}$ are fly-wheel diodes. The $V T_{1} \sim V T_{6}$ are MOSFET switches which are controlled by SPWM signals. The current in windings and the rotating speed of BSRM are adjusted by this way.

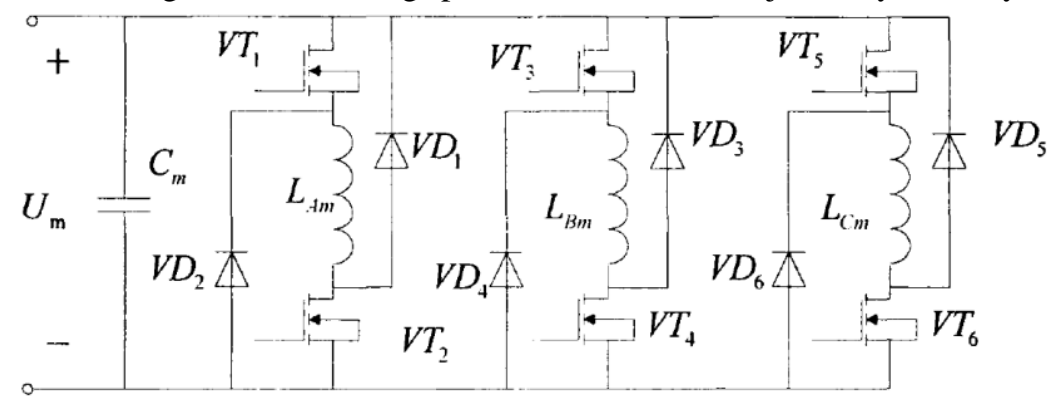

Figure 3. Diagram of the Converter Circuit

\section{Hardware Circuit Design}

The hardware circuit comprises main control unit, inverting and detecting circuit etc. In the main control unit, the MCU dsPIC30F2010 produced by Microchip is used. The dsPIC30F Motor Control family is specifically designed to control the most popular types of motors. The design takes advantage of dsPIC 30F peripherals specifically suited for motor control: Motor Control Pulse Width Modulation (MCPWM) and high-speed A/D Converter. The DSP engine of the dsPIC30F2010 supports the necessary fast mathematical operations. The dsPIC30F2010 family member is a 28-pin 16-bit DSC specifically designed for low-cost/high efficiency motor control applications. The dsPIC30F2010 provides these key features:

- 30 MIPS processing performance

- Six independent or three complementary pairs of dedicated Motor Control PWM outputs

- Six-input, 1 Msps ADC with simultaneous sampling capability from up to four inputs

- Multiple serial communications: UART, I2C ${ }^{\text {TM }}$ and SPI

- Small package (6 mm x $6 \mathrm{~mm}$ QFN) for embedded control applications

- DSP engine for fast response in control loops

The circuit of main control unit is shown in Fig. 4.

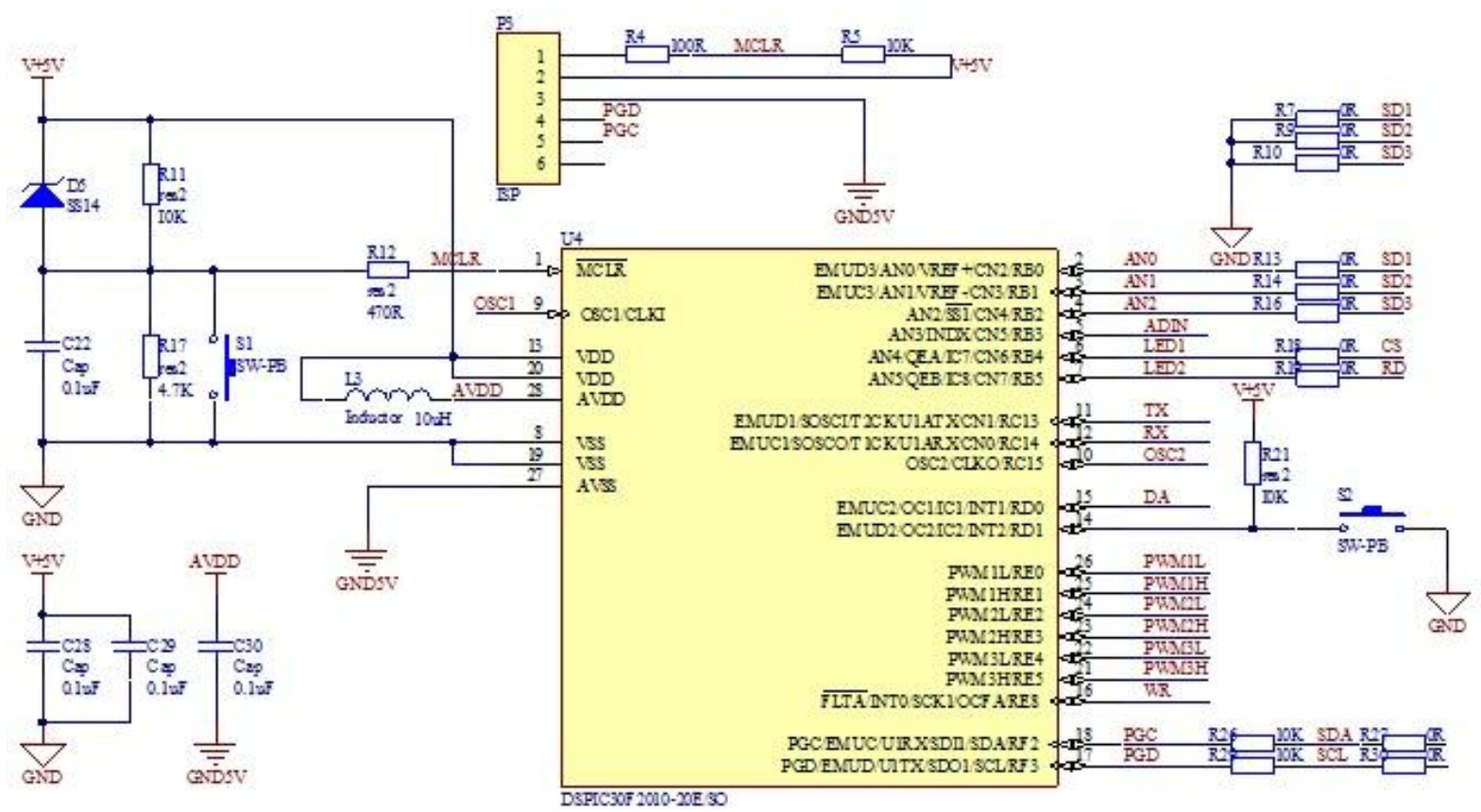

Figure 4. Circuit of Main Control Unit 
The SPWM control signals for MOSFET $V T_{1} \sim V T_{6}$ in Fig. 3 are PWM1L, PWM1H, PWM2L, PWM2H, PWM3L, PWM3H. In order to decrease the disturbance of these signals, the optocoupler 6 N136 is used for isolation. The circuit is shown in Fig. 5.

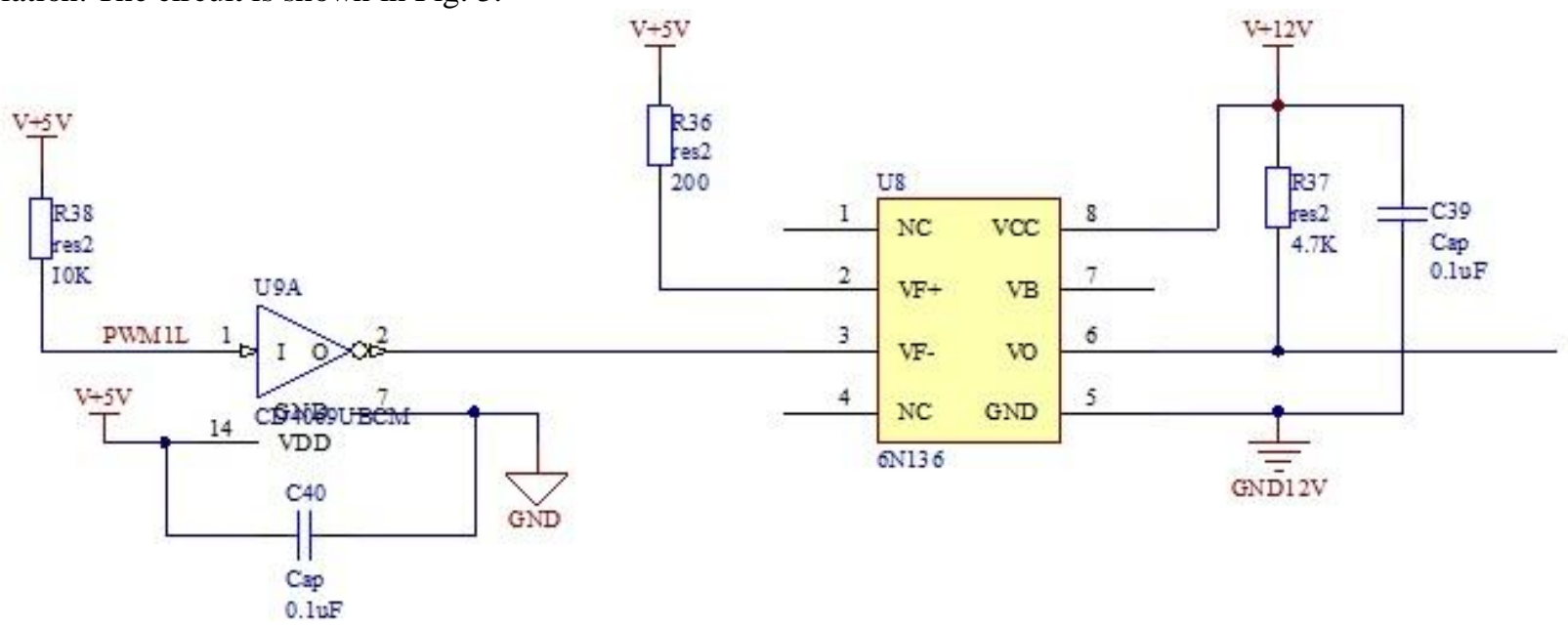

Figure 5. Circuit of Optocoupler

The IR2110 are used to drive the MOSFETs in inverting circuit. The IR2110 are high voltage, high speed power MOSFET and IGBT drivers with independent high and low side referenced output channels. Proprietary HVIC and latch immune CMOS technologies enable ruggedized monolithic construction. Logic inputs are compatible with standard CMOS or LSTTL output, down to 3.3V logic. The output drivers feature a high pulse current buffer stage designed for minimum driver cross-conduction. Propagation delays are matched to simplify use in high frequency applications. The floating channel can be used to drive an N-channel power MOSFET or IGBT in the high side configuration which operates up to 500 or 600 volts. The IR 2110 provides these key features:

- Floating channel designed for bootstrap operation

- Gate drive supply range from 10 to $20 \mathrm{~V}$

- Undervoltage lockout for both channels

-3.3V logic compatible

- CMOS Schmitt-triggered inputs with pull-down

- Cycle by cycle edge-triggered shutdown logic

- Matched propagation delay for both channels

- Outputs in phase with inputs

The inverting circuit is shown in Fig. 6.

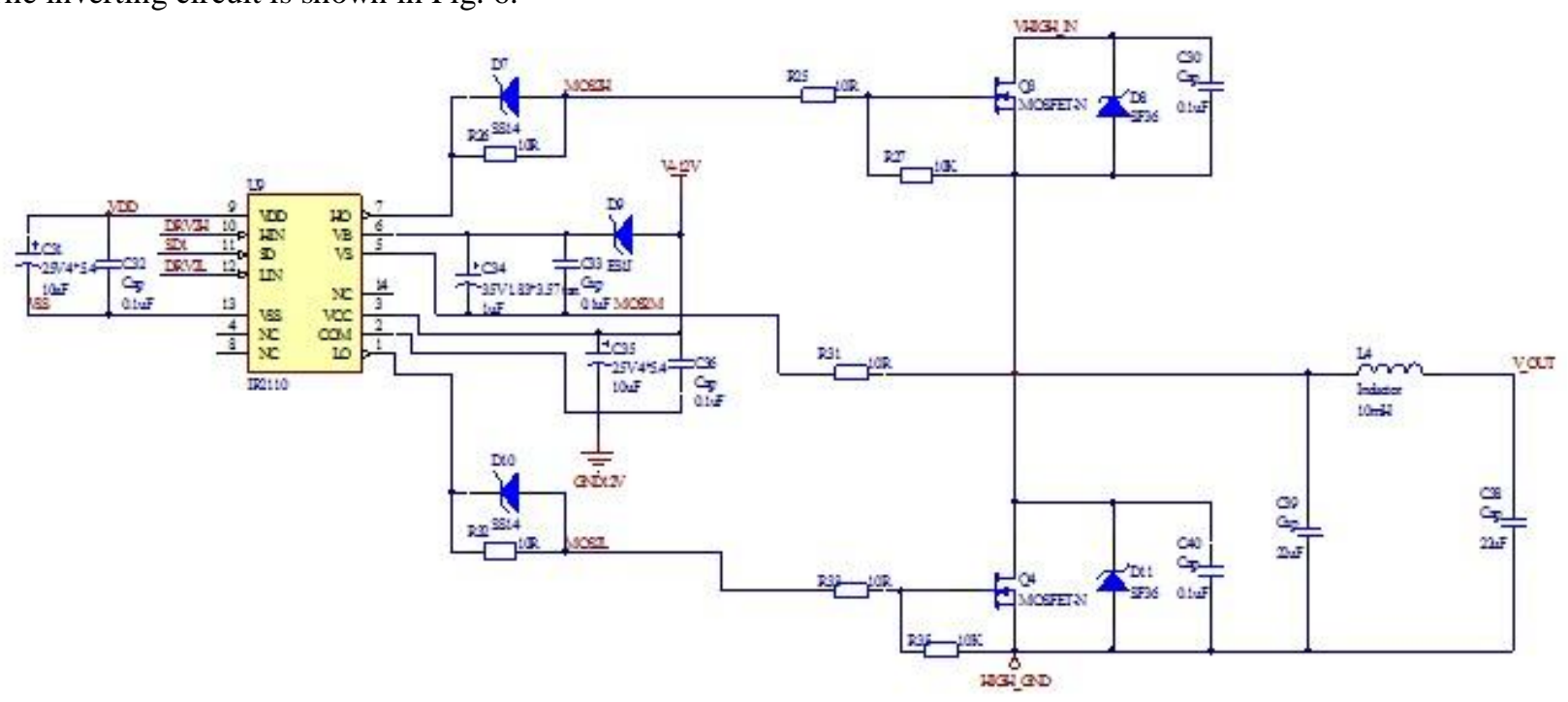

Figure 6. Inverting Circuit

The hardware circuit also include keys for inputting, LEDs for displaying, LDO for voltage regulating and current detecting for protection. The real PCB is shown in Fig. 7. 


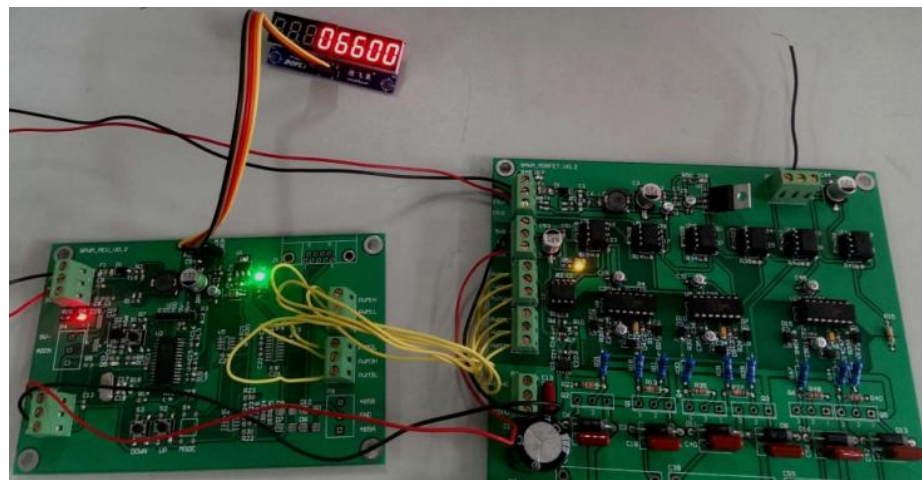

Figure 7. Real PCB

\section{Software Design}

The software of MCU is programmed by C in MPLAB and compiled by PIC30. It has several modules such as main control module, AD convert module, PWM module, LED module, UART module. The programming IDE is shown in Fig. 8.

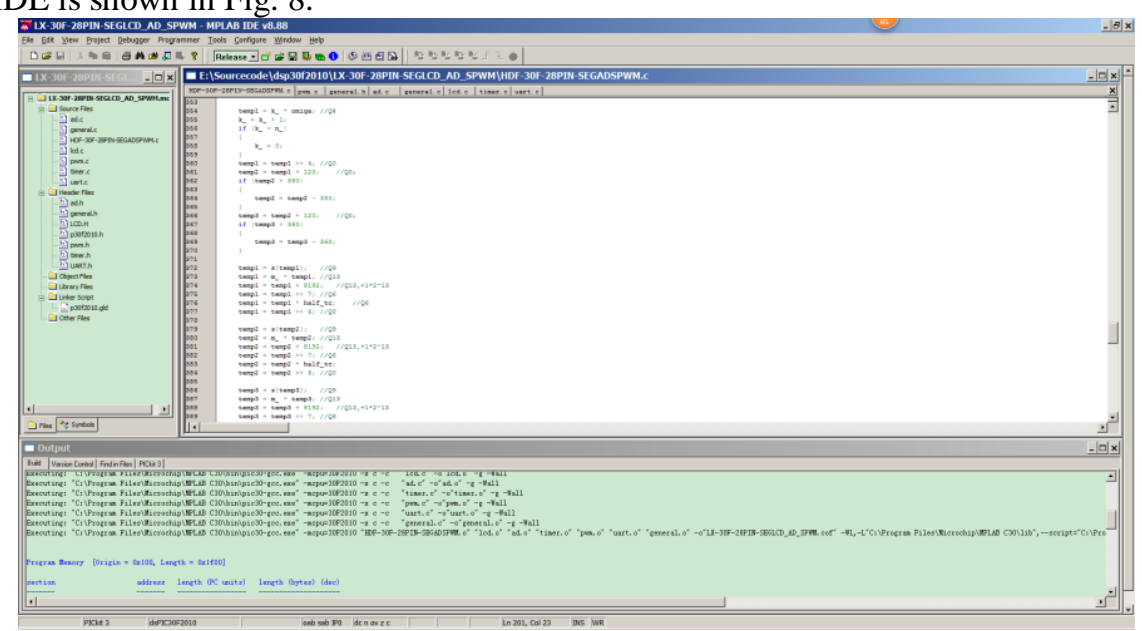

Figure 8. Programming IDE

The key part of software is how to generate SPWM signal and it is realized by calculating the position and looking up the sine table. This part is put in the interrupt service routine in order to guarantee the real-time performance. The demo code is shown in Table 1.

Table 1. Demo Code of SPWM Generating

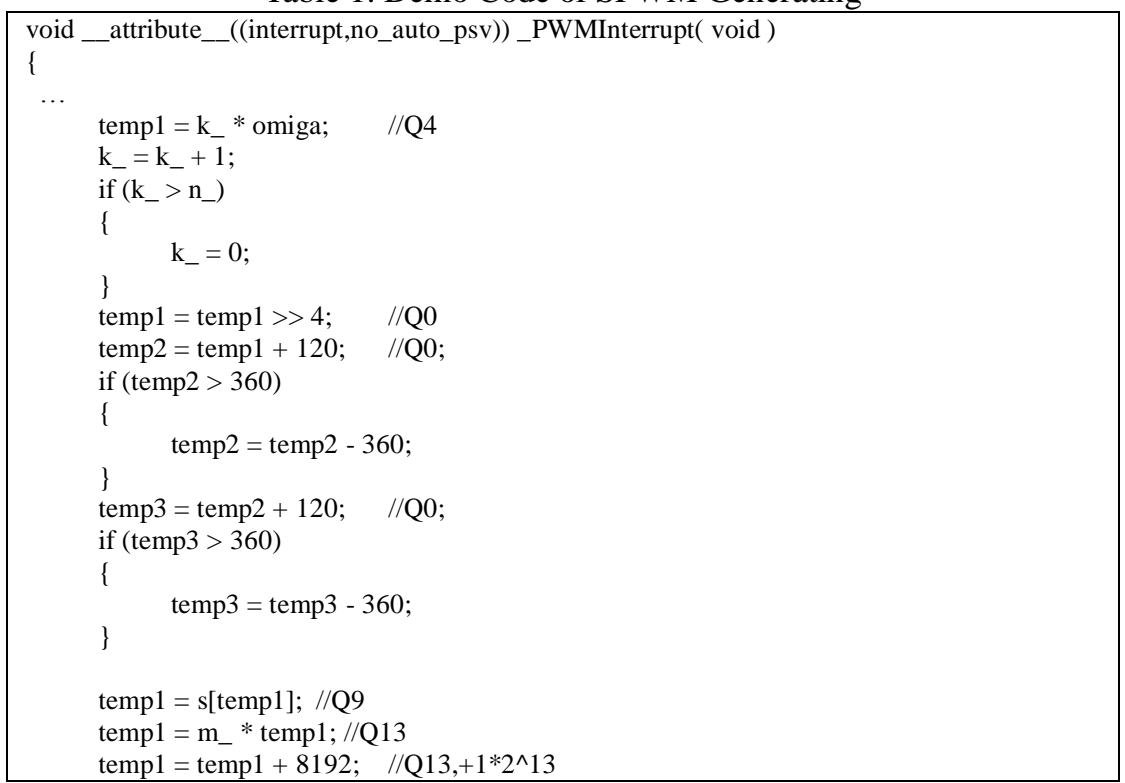




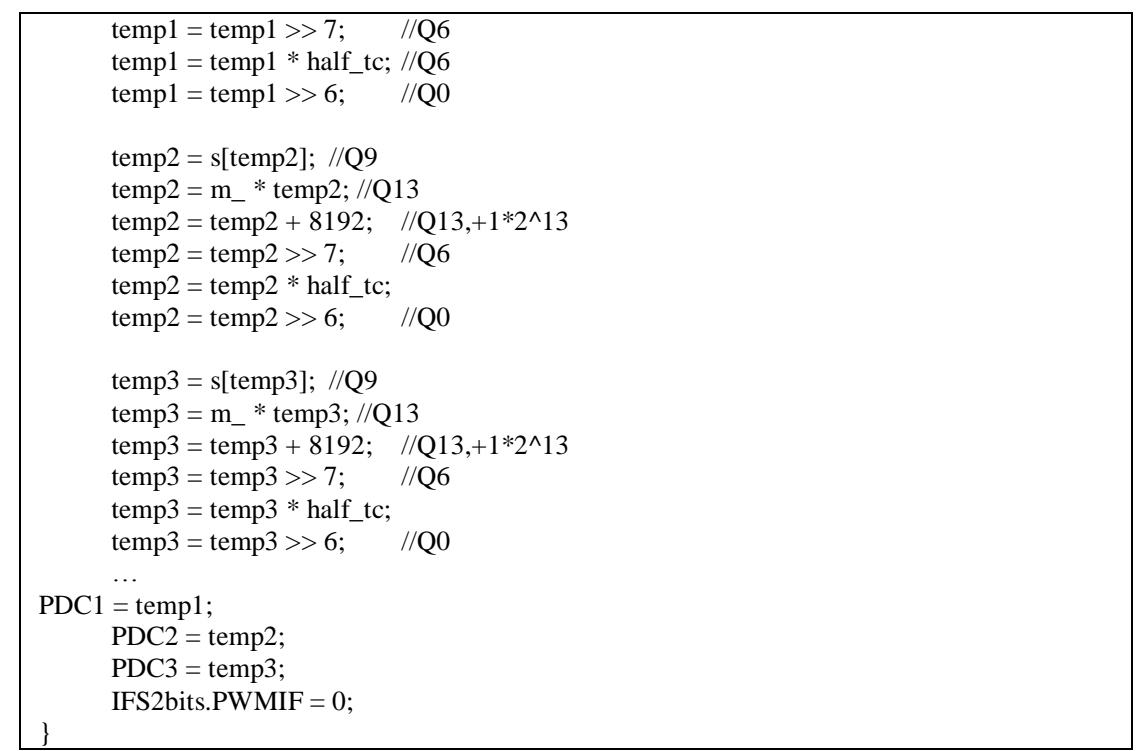

\section{Results}

The outputs of three phases are tested by oscilloscope (Agilent Technologies DSO-X 2024A). The frequency of $50 \mathrm{~Hz}, 1000 \mathrm{~Hz}$ and $5000 \mathrm{~Hz}$ signals are shown in Fig. 9 Fig. 11.

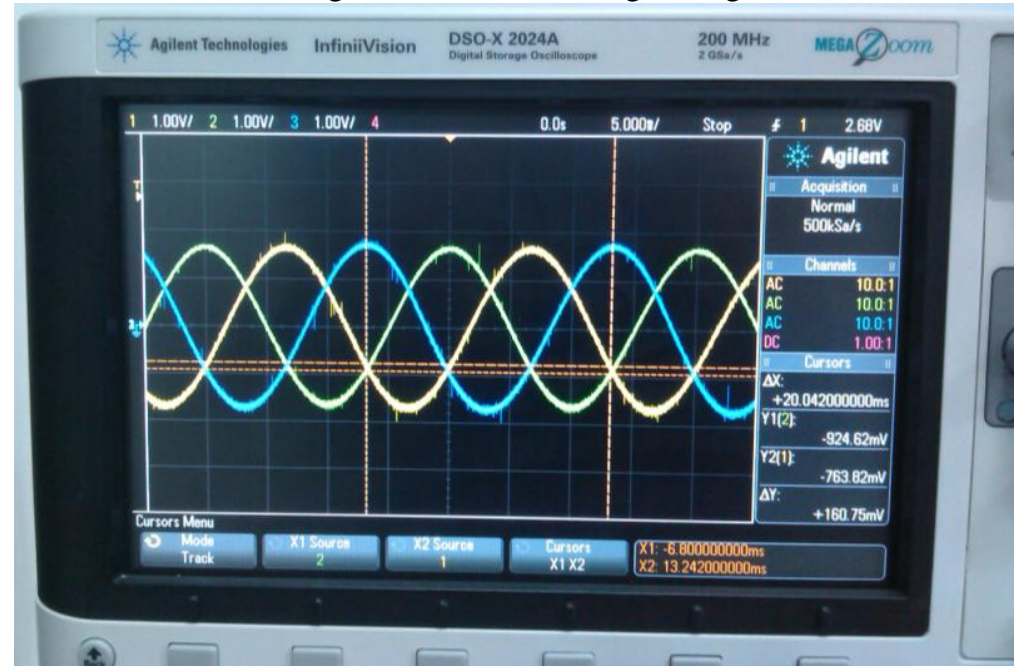

Figure 9. Output of $50 \mathrm{~Hz}$

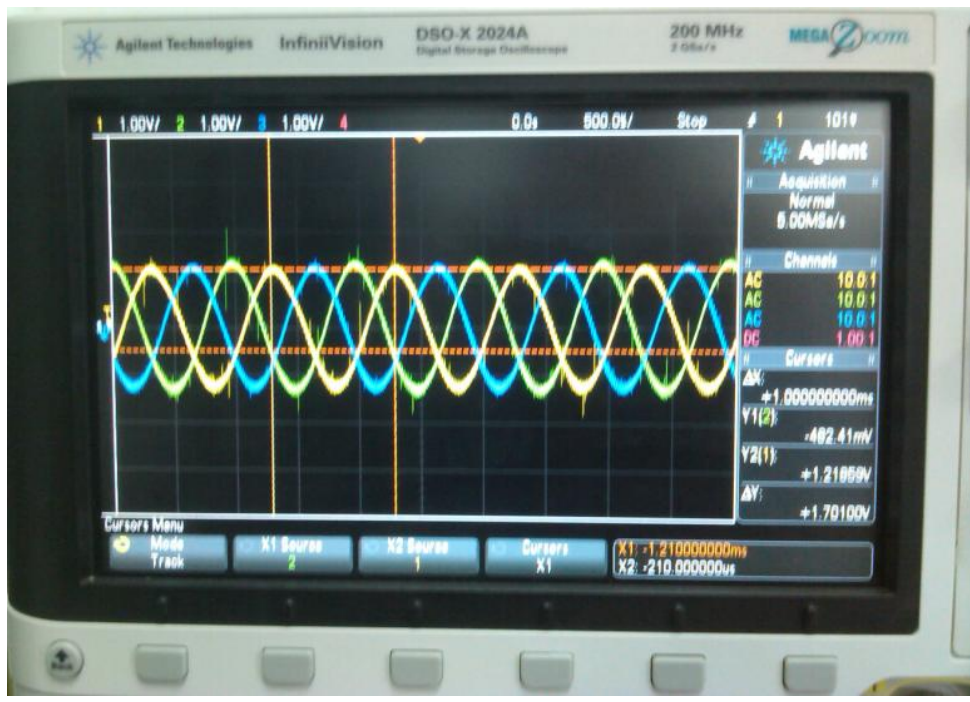

Figure 10. Output of $1000 \mathrm{~Hz}$ 


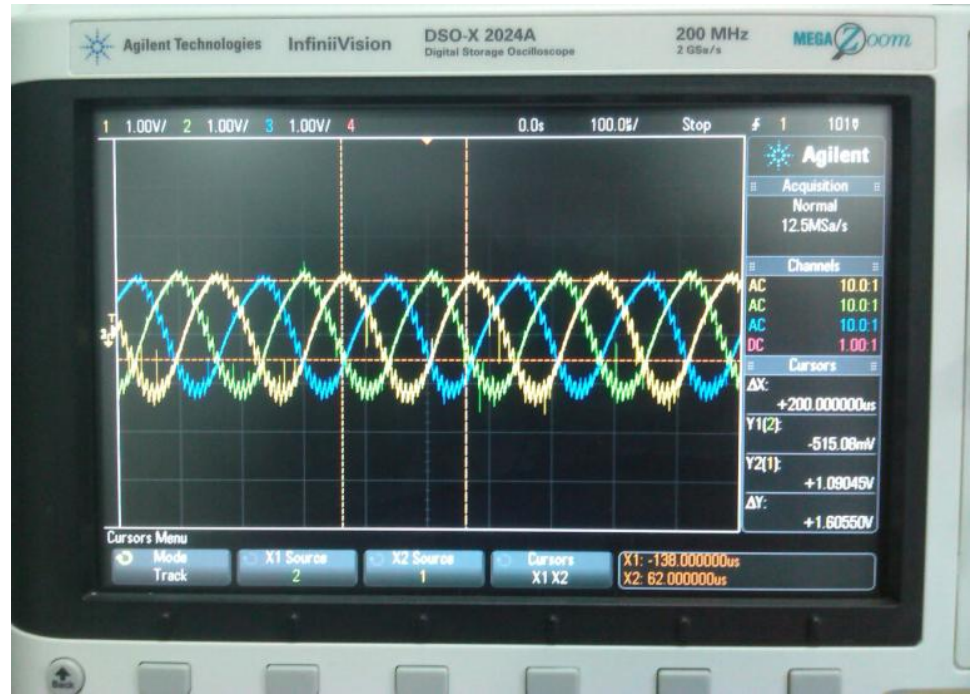

Figure 11. Output of $5000 \mathrm{~Hz}$

\section{Conclusion}

The hardware circuit and software introduced above realize the power supply for three phase bearingless switched reluctance motor based on SPWM. The three phase signals approximate sinusoid and they have $120^{\circ}$ phase difference. The frequency of output signals varies from $50 \mathrm{~Hz}$ to $10 \mathrm{kHz}$ by the aid of dsPIC30F2010's strong DSP ability. The power supply satisfies the demand of BSRM.

\section{Acknowledgement}

This work was financially supported by the College students practice and innovation training plan of Soochow University (No. 2013xj039) and National computer and information technology experiment teaching demonstration center of China.

\section{References}

[1]. M.Takemoto, H.Suzuki, A.Chiba, et al. Improved Analysis of a Bearingless Switched Reluctance Motor [J]. IEEE Transactions on Industry Applications, 2001, 37(1): 26-34.

[2]. A.Chiba, T.Deido, T.Fukao, et al. An Analysis of Bearingless AC Motors [J]. IEEE Transactions On Energy Conversion, 1994, 9(1): 61-67.

[3]. A.Chiba, D.T.Power, M.A.Ralznlan. Characteristics of a Bearingless Induction Motor [J]. IEEE Trans. On Magnetics, 1991, 27(6): 5199-5201.

[4]. A.Chiba, D.T.Power, M.A.Rahman. Analysis of No-load Characteristics of a Bearingless Induction Motor [J]. IEEE Trans. On Industry Applications, 1995, 31(1): 77-83.

[5]. A.Chiba, R.Furuichi, Y.Aikawa, et al, Stable Operation of Induction-type Bearingless Motors under Loaded Conditions [J]. IEEE Trans. On Industry Applications, 1997, 33(4): 919-924.

[6]. Zhenyu Yu, Arefeen Mohammed, Issa Panahi. A Review of Three PWM Techniques. Proceedings of the American Control Conference Albuquerque, New Mexico. 1997, pp. 257-261.

[7]. D. G. Holmes, T. A. Lipo. Pulse Width Modulation for Power Converters: Principles and Practice. M.E. El-Hawary, Ed. New Jersey: IEEE Press, Wiley- Interscience. 2003, pp. 215-313.

[8]. Wajiha Shireen, Srinivas Vanapalli, Hrishikesh Nene. A DSP Based SVPWM Control for Utility Interactive Inverters used in Alternate Energy Systems. IEEE Transactions on Industrial Electronics, 2006. 